\title{
Effect of Sample Size on the Control Limits of Exponentially Weighted Moving Average Distance Square Scheme
}

\author{
Athambawa Mohamed Razmy \\ Department of Mathematics and Statistics, Sultan Qaboos University, Muscat, Oman \\ Email:mrazmy@squ.edu.om, amrazmy@seu.ac.lk
}

Received 22 May 2016; accepted 17 June 2016; published 21 June 2016

Copyright (C) 2016 by author and OALib.

This work is licensed under the Creative Commons Attribution International License (CC BY). http://creativecommons.org/licenses/by/4.0/

(c) (i) Open Access

\begin{abstract}
In the series of quality monitoring schemes with exponentially weighted moving average, the exponentially weighted moving average distance square scheme was introduced for joint monitoring of process mean and variance. This scheme claims that it has a special feature that the control limits of the scheme are independent of sample size and therefore it gives more freedom to the users. However, this claim was not studied in detail. In this study, the control limits were found for this scheme through simulations, for different sample sizes with different combination of other scheme parameters. This study concludes that the control limits for designing this scheme are independent of sample size.
\end{abstract}

\section{Keywords}

Average Run Length, Control Limit, Exponential Weighted Moving Average, Joint Monitoring

Subject Areas: Mathematical Statistics

\section{Introduction}

The exponentially weighted moving average (EWMA) chart was introduced for monitoring the sample mean of a quality parameter by Robrts in 1959 [1]. This chart is more sensitive in detecting any small shift in mean compared to the conventional Shewhart control chart which was introduced by Shewhart in 1939 [2]. In EWMA chart, EWMA Variable $A_{t}$ is plotted against the sample number $t(t=1,2, \cdots)$, where

$$
A_{t}=\left(1-\lambda_{m}\right) A_{t-1}+\lambda_{m} \bar{X}_{t} \text {. }
$$

Normally $A_{0}$, is considered as the target mean $\mu_{0}, \lambda_{m}$ is a constant such that $0<\lambda_{m}<1$ and it is selected based on the shift in the mean to be detected quickly for any process for a given in-control average run length (ARL).

How to cite this paper: Razmy, A.M. (2016) Effect of Sample Size on the Control Limits of Exponentially Weighted Moving Average Distance Square Scheme. Open Access Library Journal, 3: e2663. http://dx.doi.org/10.4236/oalib.1102663 
$\bar{X}_{t}$ is the $t^{\text {th }}$ sample mean of the quality parameter, to be monitored. This chart issues an out-of-control signal if $A_{t}$ is greater than the upper control limit (UCL) or lower than the lower control limit (LCL). For designing this chart, $\lambda_{m}$ values and control limits for detecting different shifts in mean under different sample sizes and incontrol ARLs, can be found in Crowder (1989) [3].

Chang and Gan introduced an EWMA chart for monitoring sample variance of a quality parameter in 1993 [4]. In their chart, the EWMA variable $B_{t}$ is plotted against the sample number $t(t=1,2, \cdots)$, where

$$
B_{t}=\left(1-\lambda_{v}\right) B_{t-1}+\lambda_{v} \log \left(S_{t}^{2}\right) .
$$

Normally, the value of $B_{t}$ is taken as $E\left[\log \left(S_{t}^{2}\right)\right]$ and $S_{t}^{2}$ is the sample variance of the quality parameter interested. $\lambda_{v}$ is a positive constant which has the possible values of $0<\lambda_{v}<1$ and it is based on the shift in the variance to be detected quickly for a given in-control ARL. Like in the EWMA chart for monitoring sample mean, this chart also issues an out-of-control signal if $B_{t}$ is greater than UCL or the lower than the LCL.

The above discussed two EWMA charts are used for monitoring the process mean and variance independently. In 1997, it was understood that monitoring the sample mean and variance was a bivariate problem and these two had to be monitored jointly [5]. A joint monitoring scheme for monitoring mean and variance simultaneously using EWMA technique was introduced by Gan [6]. Another two joint monitoring schemes, called max EWMA scheme and EWMA semicircle scheme for joint monitoring of process mean and variance were proposed by Chen et al. [7] [8]. The scheme parameters to design these joint monitoring schemes differ based on the sample sizes. This issue restricts the users in selecting a convenient sample size.

Subsequently, a new joint monitoring scheme named EWMA distance square scheme (EWMAD2) was introduced with a claim that its control limits were independent of sample size [9]. This scheme uses the standardized sample mean $U_{t}$ and variance $V_{t}$ such that

$$
U_{t}=\frac{\bar{X}_{t}-\mu_{0}}{\sigma_{0} / \sqrt{n}}
$$

and

$$
\begin{gathered}
V_{t}=\Phi^{-1}\left[H\left(\frac{(n-1) S_{t}^{2}}{\sigma_{0}^{2}}: n-1\right)\right] . \\
H\left[\frac{(n-1) S_{t}^{2}}{\sigma_{0}^{2}}: n-1\right]=H(w ; v)=P(W \leq w) \text { for } W \sim \chi_{v}^{2}
\end{gathered}
$$

the chi-square distribution with $v$ degrees of freedom and $\phi($.$) is the cumulative distribution function of a stan-$ dard normal random variable [10]. $S_{t}$ is the sample standard deviation, $\sigma_{0}$ is the population standard deviation and $n$ is the sample size.

A statistics $D_{t}^{2}$ is defined by

$$
D_{t}^{2}=U_{t}^{2}+V_{t}^{2}
$$

The EWMAD2 scheme is obtained by plotting the EWMAD2 statistic $C_{t}$ against the sample number $t$ where

$$
C_{t}=\left(1-\lambda_{d}\right) C_{t-1}+\lambda_{d} D_{t}^{2} .
$$

$C_{0}=E\left(D_{t}^{2}\right)=0$ and $\lambda_{d}$ is a constant selected based on the shift in $D_{t}^{2}$, to be detected quickly. The optimum $\lambda_{d}$ values for detecting various shifts in $D_{t}^{2}$, for the selected in-control ARLs can be read in Razmy (2005) [9]. This research paper examines the effect of sample size on the control limits of the EWMAD2 scheme for different ARLs.

\section{Methodology}

For this study, commonly used in-control ARLS100, 250, 300, 370, 500 and 1000 were selected. The sample sizes $(n)$ studied were 5, 10, 50, 100 and 150. Samples were simulated in SAS using proc RANNO with sample sizes $n$. For each sample, the statistics $D_{t}^{2}$ was calculated from the variables $u_{t}$ and $v_{t}$. The statics $C_{t}$ was ob- 
tained for different values of $\lambda_{d}\left(\lambda_{d}=0.05,0.1,0.2, \cdots, 0.9,1.0\right)$.

Initially arbitrary control limits $(C L)$ were assumed for each combination of $\lambda_{d}$ and in-control ARLs. Then, if $C_{1}<C L$, then the second sample was simulated. This procedure was continued till $C_{t}>C L$ where $C_{t}$ is an out-of-control point. Each time, the number of samples generated till to find an out-of-control point was recorded and it is the run length. In the same way 100,000 runs were performed and the ARLs were found. Subsequently, the assumed $C L$ values were adjusted till to obtain the required in-control ARLS of 100, 250, 300, 370, 500 and 1000 for different $\lambda_{d}$ values. By this procedure, the CLs for different combinations of in-control ARLs, sample sizes and $\lambda_{d}$ values were found. In all cases, simulations were run until the standard error of the ARL was less than $1 \%$ of the pre-specified ARL.A sample program with sample size 5, is given in the Appendix.

\section{Results and Discussion}

The obtained CLs were plotted against the lambda value for the selected in-control ARLS and sample sizes. From the Figures 1-5, it could be observed that the control limits was increased with $\lambda_{d}$. It is obvious that the $C L$ increases with in-control ARLs for a given $\lambda_{d}$ value. The main observation is, for given $\lambda_{d}$ and in-control ARL, the $C L$ limit is constant with any sample size. This proves the claim that in the EWMAD2 scheme, $C L s$ are independent of sample size and this property eases the procedure of designing this scheme with unrestricted sample sizes.

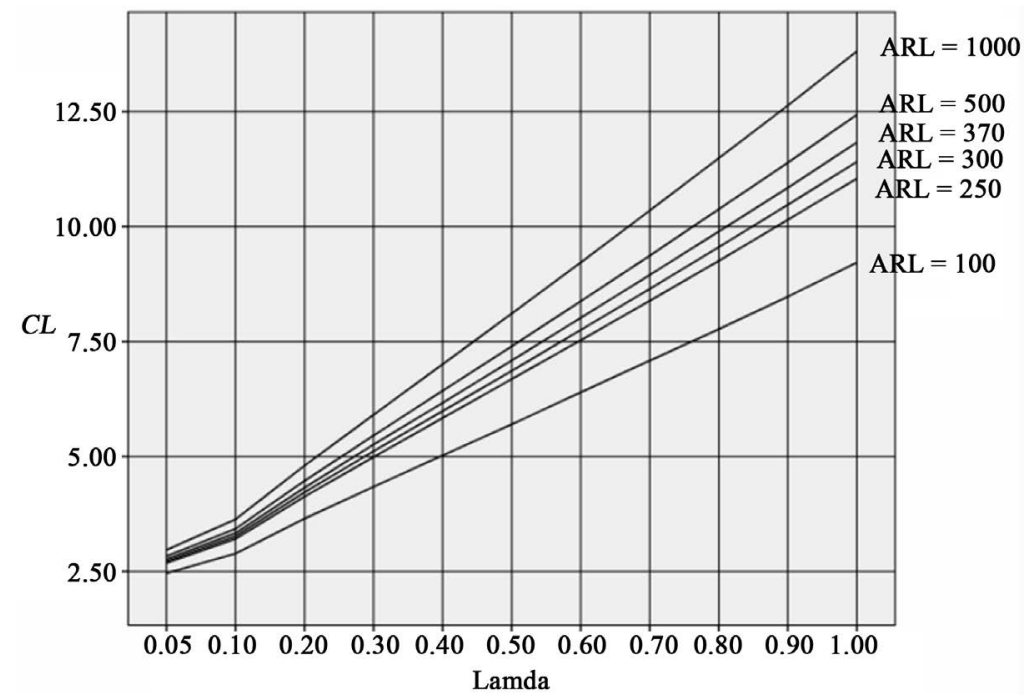

Figure 1. Control limits for selected in-control ARLS, $n=5$.

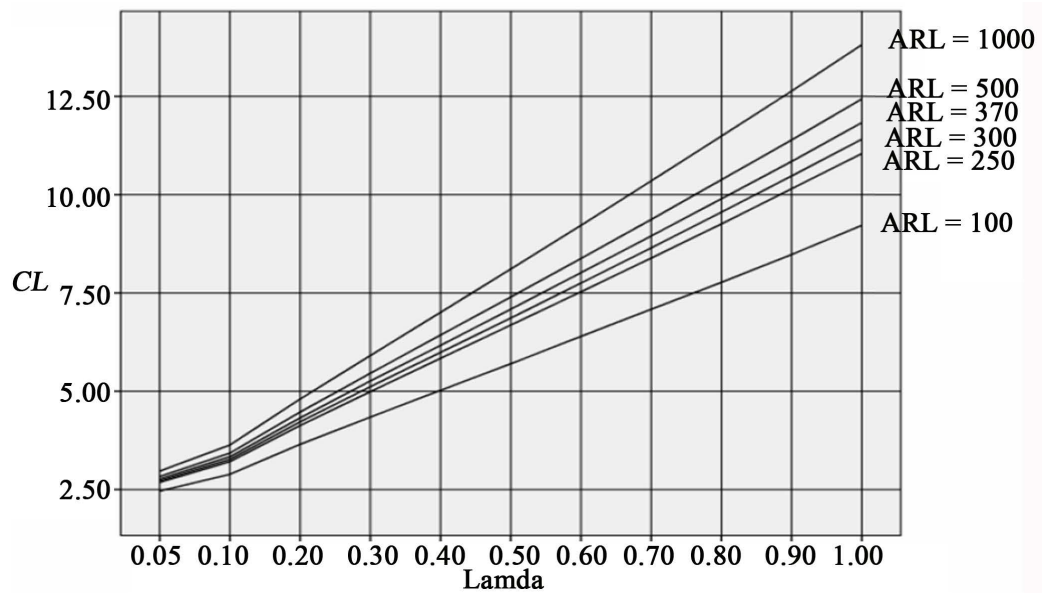

Figure 2. Control limits for selected in-control ARLS, $n=10$. 


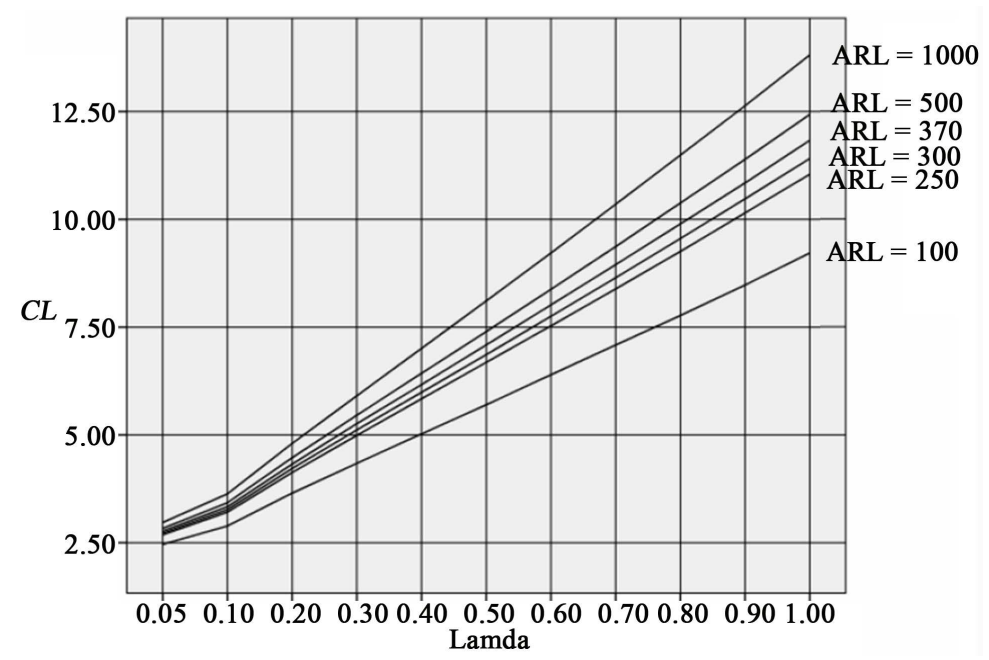

Figure 3. Control limits for selected in-control ARLS, $n=50$.

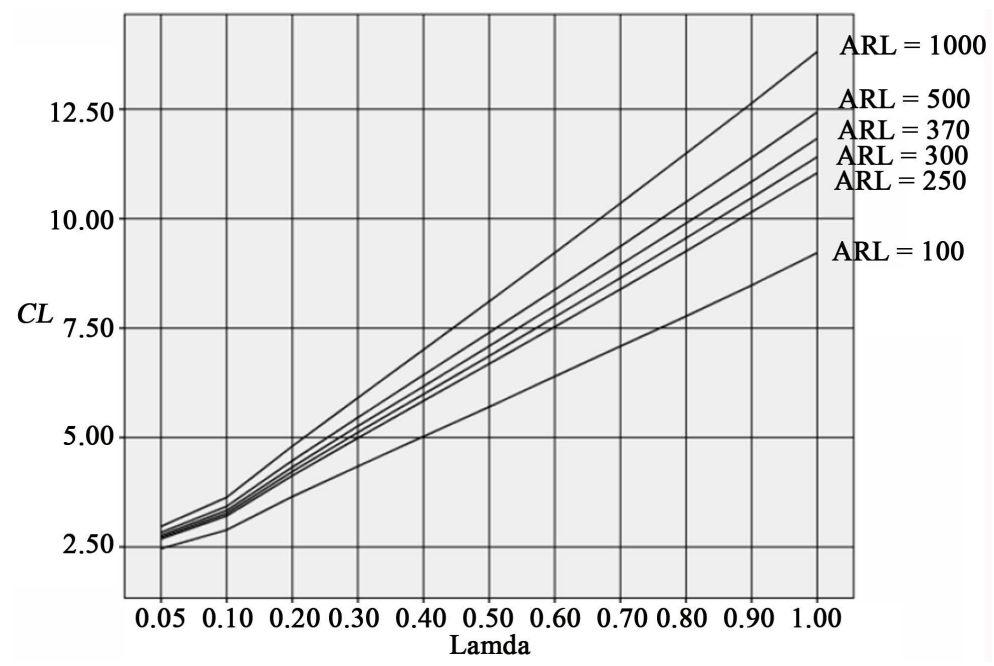

Figure 4. Control limits for selected in-control ARLS, $n=100$.

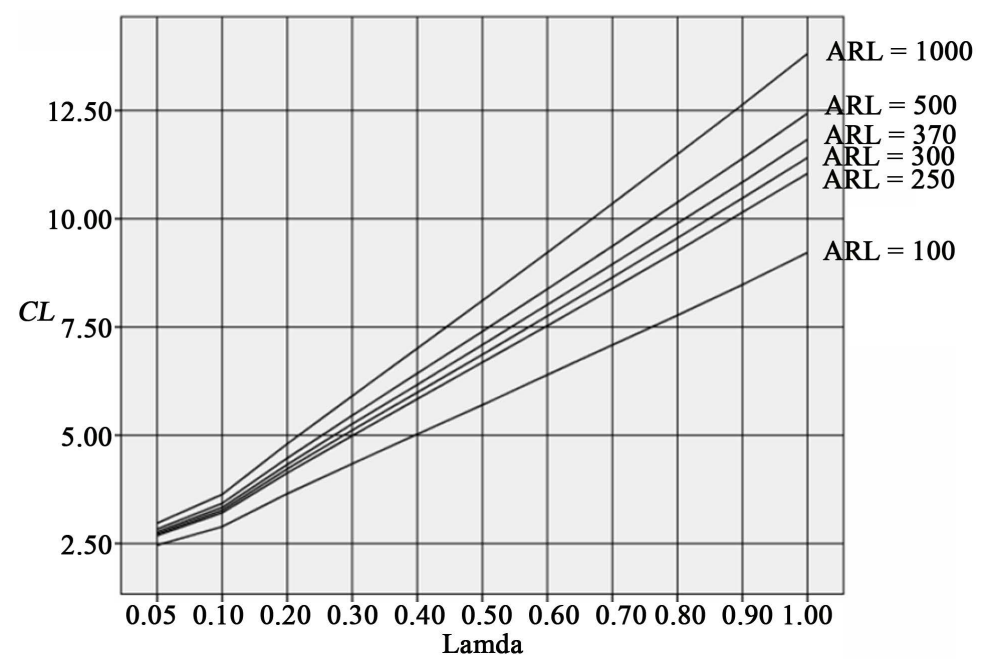

Figure 5. Control limits for selected in-control ARLS, $n=150$. 


\section{Acknowledgements}

The outputs for numerous programs were recorded and arranged by Ms. Iresha Dilhani.

\section{References}

[1] Roberts, S.W. (1959) Control Chart Tests Based on Geometric Moving Averages. Technometrics, 1, 239-250. http://dx.doi.org/10.1080/00401706.1959.10489860

[2] Shewhart, W. A. (1939) Statistical Methods from the Viewpoint of Quality Control. Graduate School, Department of Agriculture, Washington DC, 75.

[3] Crowder, S.V. (1989) Design of Exponentially Weighted Moving Average Schemes. Journal of Quality Technology, 21, 155-162.

[4] Chang, T.C. and Gan, F.F. (1993) Optimal Designs of One-Sided EWMA Charts for Monitoring a Process Variance. Journal of Statistical Computing \& Simulations, 49, 33-48. http://dx.doi.org/10.1080/00949659408811559

[5] Gan, F.F. (1997) Joint Monitoring of Process Mean and Variance. Nonlinear Analysis, Theory, Methods and Applications, 30, 4017-4024. http://dx.doi.org/10.1016/S0362-546X(97)00224-1

[6] Gan, F.F. (1995) Joint Monitoring of Process Mean and Variance Using Exponentially Weighted Moving Average Control Charts. Technometrics, 37, 446-453. http://dx.doi.org/10.1080/00401706.1995.10484377

[7] Chen, G., Cheng, S.W. and Xie, H.W. (2001) Monitoring Process Mean and Variability with One EWMA Chart. Journal of Quality Technology, 33, 223-233.

[8] Chen, G., Cheng, S.W. and Xie, H.W. (2004) A New EWMA Control Chart for Monitoring Both Location and Dispersion. Quality Technology \&Quantitative Management, 1, 217-231. http://dx.doi.org/10.1080/16843703.2004.11673074

[9] Razmy, A.M. (2005) Joint Monitoring of Process Mean and Variance. MSc Thesis, Department of Statistics and Applied Probability, National University of Singapore, Singapore.

[10] Quesenberry, C.P. (1995) On Properties of Q Charts Variables. Journal of Quality Technology, 21, 242-250. 


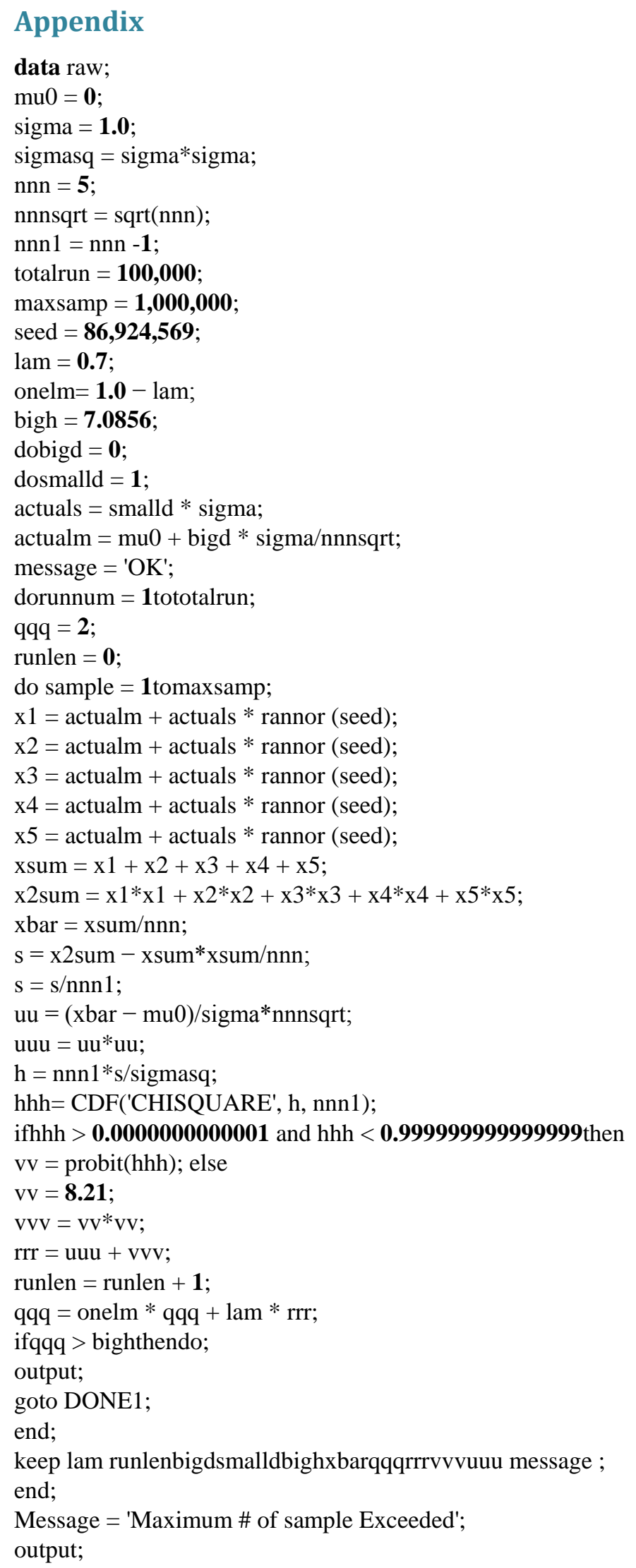


DONE1:

end;

end;

end;

procmeans data $=$ raw;

by bigdsmalld;

run; 